\title{
Frontal Sinus Fracture: A Case Report
}

\author{
Anurag Singh • Vikrant Bhardwaj • \\ S. Sharma
}

Received: 16 November 2010/Accepted: 17 November 2010/Published online: 10 May 2011

(C) Association of Oral and Maxillofacial Surgeons of India 2011

\begin{abstract}
In today's world high velocity trauma is not uncommon and most of the road traffic accidents involve trauma to head and neck region. For purposes of initial evaluation, Injury to the frontal bone should be regarded as head injuries. The majority of these patients are victims of automobile accidents or fall from height with multiple injuries requiring a multispecialty team approach and complete evaluation. The standard trauma protocol must be followed with emphasis on ensuring an adequate airway, breathing, circulation, central nervous system status, and cervical-spine. Any other life-threatening injuries take precedence over the sinus fracture. Management of a case of frontal sinus fracture with loss of anterior table is presented.
\end{abstract}

Keywords Frontal sinus - Frontonasal duct - Anterior and posterior table $\cdot$ Paraumbilical fat $\cdot$ Iliac crest graft

\section{Introduction}

Fractures involving the frontal region represent some of the least common injuries that affect the facial skeleton. The

Comment Treatment of frontal sinus fractures has been controversial for years due to the relatively small number of patients and short period of follow up. The goals, however, remain the same: cosmetic restoration and prevention of early and late complications including acute and chronic sinusitis, mucocele formation, brain abscess, and osteomyelitis [3]. The first step in the treatment of frontal sinus fractures is to evaluate and manage more serious life threatening injuries.

A. Singh $(\bowtie) \cdot$ V. Bhardwaj $\cdot$ S. Sharma

Department of Burns, Plastic and Maxillofacial Surgery,

Safdarjung Hospital, New Delhi 110029, India

e-mail: dranurag_in@yahoo.com incidence is $5-15 \%$ of all facial fractures [5]. However, because the frontal area makes up part of the brain case, the fatal potential of these injuries is much greater than with other facial fractures. A thorough neurologic examination is extremely important.

Significant intracranial injury occurs more commonly with injury to the frontal sinus (12-17\% of the time) than with injury to the mandible or mid face due to the proximity of the frontal sinus to the brain and the great forces required to cause a frontal sinus fracture [1]. Neurosurgical consultation should be obtained promptly if abnormal neurologic studies or brain CT changes are observed. Changes in mental status as well as nausea or vomiting are suggestive of intracranial injury. It is important to search for cerebrospinal fluid leakage from the nose or directly from lacerations and exposed bone which could signify dural displacement coupled with a posterior table fracture [2] (Figs. 1, 2). A traumatic CSF fistula of the frontal sinus is a life threatening process deserving urgent attention [2].

\section{Case Report}

A 22-year-old male sustained injury to frontal bone following road traffic accident was referred to us from rural hospital (Fig. 3) after 2 days of sustaining injury. Patient lost consciousness immediately after sustaining trauma and was admitted in some rural hospital for 2 days following which he was referred to Safdarjung Hospital.

Patient was conscious and well oriented at the time of presentation but had a positive history of loosing consciousness for around half an hour.

On examination there was open fracture of the frontal bone in the midline with loss of outer table. The defect was $3 \times 2 \mathrm{~cm}$ in size with the frontal sinus directly open to 


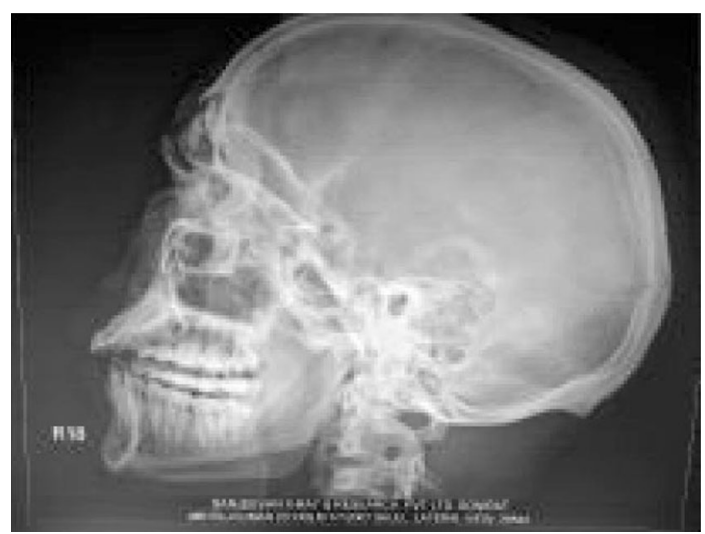

Fig. 1 Lateral skull radiograph

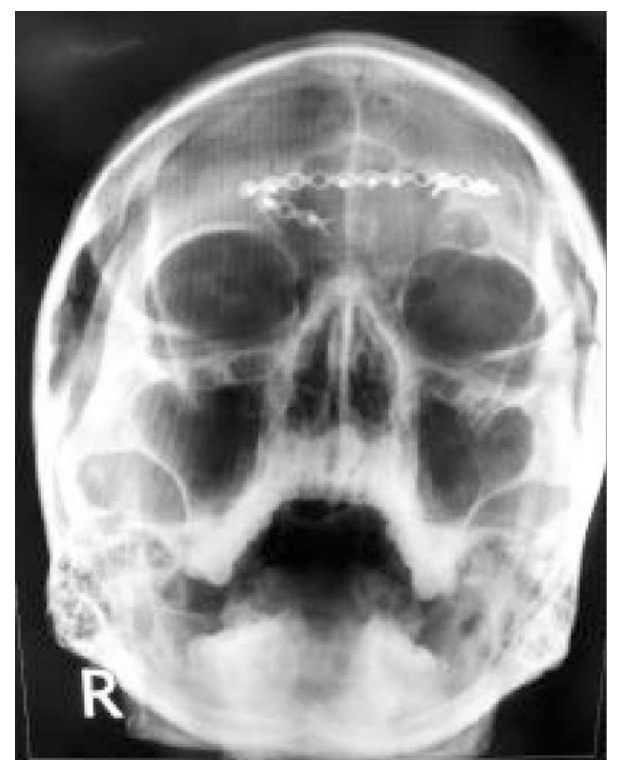

Fig. 2 Post operative PNS radiograph

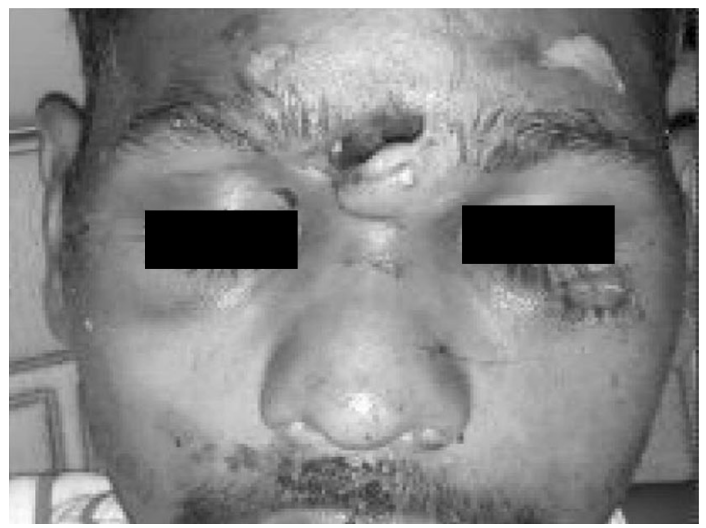

Fig. 3 Pre-operative frontal view

external environment (Fig. 3). Computed tomography revealed fracture of outer and inner table of the frontal sinus in the midline with pneumocephalus for which

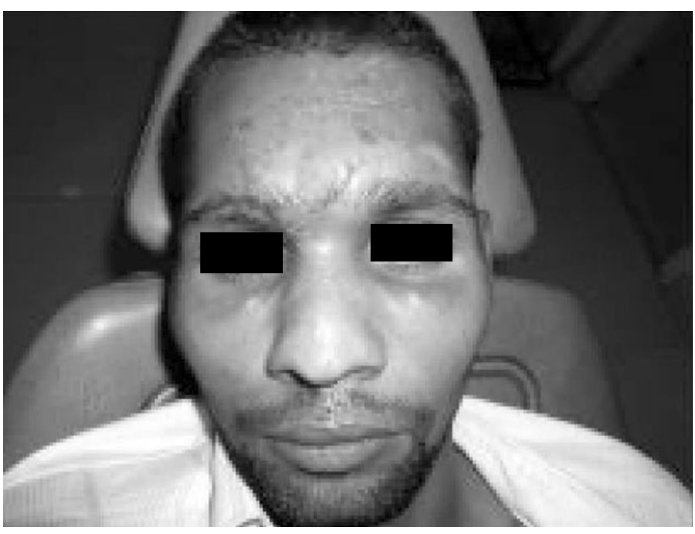

Fig. 4 Post operative frontal view

patient was kept under neurosurgical observation for $72 \mathrm{~h}$. After neurosurgical clearance and pre-anesthetic consultation patient was taken for surgery under general anesthesia with oral endotracheal intubation. After achieving asepsis the entire lining of the frontal sinus was removed. Frontonasal duct was obliterated using bone from iliac crest and the entire frontal sinus was filled with fat harvested from paraumbilical region. The lost outer table was reconstructed using bone from iliac crest and fixed with mini plates. Soft tissue was closed using primary sutures without raising any flap.

Post operative recovery was uneventful with no signs and symptoms of neurological deficits for a period of 4 months (Fig. 4), however the patient will be followed up. Once this has been accomplished, the following data is obtained in order to formulate a treatment plan: the specific bony tables involved, the type of fracture(s), presence or absence of nasofrontal duct involvement, and CSF leakage [4].

Although a treatment plan may be formed on the basis of pre-op evaluation and CT scan findings, the surgeon must be ready to modify this plan based on the findings at the time of the exploration. A risk to benefit evaluation must be made to determine the least operative intervention that will achieve the optimal result. This is particularly important to remember in dealing with frontal sinus fractures in children in order to avoid interfering with the growth centers and causing iatrogenically induced deformities. Definitive management of facial fractures should be delayed until the patient is stable and optimum conditions exist for a good surgical result and recovery. Frontal sinus fractures do not require immediate surgical attention unless they are associated with a neurosurgical or ophthalmologic emergency. Patients not requiring operative treatment for associated injuries should be observed for head trauma for $48 \mathrm{~h}$ prior to frontal sinus exploration. Obliteration of the frontal sinus with fat grafts through a frontal osteoplastic flap in patients with chronic sinusitis and mucocele 
formation has been applied by some to the treatment of frontal sinus fractures [3].

\section{References}

1. Duvall AJ et al (1987) Frontal sinus fractures: analysis of treatment results. Arch Otolaryngol Head Neck Surg 113:933-935
2. Donald PT, Bernstein L (1978) Compound frontal sinus injury with intracranial penetration. Laryngoscope 88:225-232

3. Bosley WR (1972) Osteoplastic obliteration of the frontal sinus laryngoscope. Laryngoscope 82:1463-1476

4. Stanley RB, Becker TS (1987) Injury of the nasofrontal orifices in frontal sinus fractures. Laryngoscope 97:728-731

5. Cummings CW (1993) Otolaryngology—head and neck surgery, 2nd edn. Mosby Year Book, St. Louis, pp 374-402 